\title{
Modification of indium-tin oxide films by alcanethiol and fatty acid self-assembled monolayers: A comparative study
}

\author{
Nadia Karsi, Philippe Lang, Mohamed Chehimi, Michel Delamar, Gilles Horowitz*
}

ITODYS, CNRS-UMR 7086, Université Denis-Diderot, 1 rue Guy de la Brosse, 75005 Paris,

France

\section{Supporting information}

\section{Orientation analysis of the IR data}

The system of coordinates used to locate the alkyl chain grafted on a surface is given in Fig. 5 . $(x, y)$ corresponds to the substrate, and the long axis of the molecule is in the $(y, z)$ plane. The long axis of the molecule is along the $u$ axis and is tilted by an angle $\alpha$ with respect to the $z$ axis, while $\beta$ corresponds to the dihedral angle between the plane of the C-C-C backbone and the $(y, z)$ plane. In conventional (isotropic) IR absorption (e.g., $\mathrm{KBr}$ pellet) the absorbance $A_{i}$ of a vibrational mode $i$ is proportional to the square of the transition dipole moment

$$
I_{i} \propto M_{i}^{2}
$$

IRRAS

In IRRAS, only the component of the transition dipole moment perpendicular to the substrate is IR active, and the intensity of mode $i$ is now given by

$$
I_{i} \propto M_{i z}^{2}
$$

* Corresponding author. E-mail address: horowitz@paris7.jussieu.fr 
$M_{i z}$ is connected to the projections of the transition dipole moment on the three axes of the $(u, v, w)$ coordinate system. If $a_{i}, b_{i}$ and $c_{i}$ are the cosines of the transition dipole moment in the $(u, v, w)$ coordinate system, $M_{i z}$ can be expressed as

$$
M_{i z}=F_{i}(\alpha, \beta) M_{i}
$$

where the angular function $F_{i}$ is given by

$$
F_{i}(\alpha, \beta)=a_{i} \cos \alpha+b_{i} \sin \alpha \cos \beta+c_{i} \sin \alpha \sin \beta
$$

If we now consider two different vibration modes $i$ and $j$, the ratio between the corresponding intensity is given by

$$
\frac{I_{i}}{I_{j}}=\left[\frac{F_{i}(\alpha, \beta)}{F_{j}(\alpha, \beta)}\right]^{2} \frac{A_{i}}{A_{j}}
$$

The angular functions of the vibrational modes of an alkyl chain are shown in Table $\mathrm{S} 1 . \delta$ is the angle between a $\mathrm{C}-\mathrm{C}$ bond and the long axis of the molecule $\left(\delta=\left(180^{\circ}-109^{\circ}\right) / 2=35.5^{\circ}\right)$.

Table S1. Angular function of the vibrational modes used in this work.

\begin{tabular}{ll}
\hline Mode & $F_{i}(\alpha, \beta)$ \\
\hline$v_{\mathrm{a}}\left(\mathrm{CH}_{3}\right)$ in plane & $\cos \alpha \sin \delta-\sin \alpha \cos \beta \cos \delta$ \\
$v_{\mathrm{a}}\left(\mathrm{CH}_{3}\right)$ out of plane & $-\sin \alpha \sin \beta$ \\
$v_{\mathrm{s}}\left(\mathrm{CH}_{3}\right)$ & $\cos \alpha \cos \delta+\sin \alpha \cos \beta \sin \delta$ \\
$v_{\mathrm{a}}\left(\mathrm{CH}_{2}\right)$ & $\sin \alpha \sin \beta$ \\
$v_{\mathrm{s}}\left(\mathrm{CH}_{2}\right)$ & $\sin \alpha \cos \beta$ \\
\hline
\end{tabular}

The rotation angle $\beta$ is obtained from the modes $v_{\mathrm{a}}\left(\mathrm{CH}_{2}\right)$ and $v_{\mathrm{s}}\left(\mathrm{CH}_{2}\right)$. Making use of Eq. (5) we get after some manipulation: 


$$
\beta=\tan ^{-1} \sqrt{\frac{I\left(v_{a} \mathrm{CH}_{2}\right) \cdot A\left(v_{s} \mathrm{CH}_{2}\right)}{I\left(v_{s} \mathrm{CH}_{2}\right) \cdot A\left(v_{a} \mathrm{CH}_{2}\right)}}
$$

For the tilt angle $\alpha$ we use the modes $v_{a}\left(\mathrm{CH}_{3}\right)$ and $v_{\mathrm{s}}\left(\mathrm{CH}_{2}\right)$. To account for the degeneracy of the former (in plane and out of plane) the angular function is taken as

$$
\mid F\left(\left.v_{a} \mathrm{CH}_{3}\right|^{2}=\frac{1}{2}\left[(\cos \alpha \sin \delta+\sin \alpha \cos \beta \cos \delta)^{2}+(\sin \alpha \sin \beta)^{2}\right]\right.
$$

Making use of Eqs. (S5) and (S7) we finally obtain

$$
\alpha=\cot ^{-1}\left[\frac{\cos \beta}{\sin \delta}\left( \pm \sqrt{\frac{2 I\left(v_{a} \mathrm{CH}_{3}\right) \cdot A\left(v_{s} \mathrm{CH}_{2}\right)}{I\left(v_{s} \mathrm{CH}_{2}\right) \cdot A\left(v_{a} \mathrm{CH}_{3}\right)}-\tan ^{2} \beta}-\cos \delta\right)\right]
$$

\section{Normal incidence absorption}

Under normal incidence, the electric field is within the $(x, y)$ plane. In this case, the absorbance is proportional to the square of the projection $M_{x y}$ of the transition dipole moment on the $(x, y)$ plane. Because $M_{x y}^{2}=M^{2}-M_{z}^{2}$, the relevant equation is derived by replacing $F^{2}$ by $1-F^{2}$ in Eq. (S5). Using the same vibration mode as above, the equation comes

$$
\frac{I\left(v_{a} \mathrm{CH}_{3}\right)}{I\left(v_{s} \mathrm{CH}_{2}\right)}=\frac{1-\frac{1}{2}\left[(\cos \alpha \cos \delta-\sin \alpha \cos \beta \cos \delta)^{2}+(\sin \alpha \sin \beta)^{2}\right]}{1-(\sin \alpha \cos \beta)^{2}} \cdot \frac{A\left(v_{a} \mathrm{CH}_{3}\right)}{A\left(v_{s} \mathrm{CH}_{2}\right)}
$$

Eq. (S9) has no analytical solution, so the tilt angle $\alpha$ was estimated through a numerical calculation. 